\title{
Precision surgery with the genetic assessment for operable pancreatic cancer beyond the radiological assessment alone
}

\author{
Hiromitsu Hayashi, Hideo Baba \\ Department of Gastroenterological Surgery, Graduate School of Life Sciences, Kumamoto University, Kumamoto, Japan \\ Correspondence to: Hideo Baba, MD, PhD, FACS. Department of Gastroenterological Surgery, Graduate School of Life Sciences, Kumamoto \\ University, 1-1-1 Honjo, Chuo-ku, Kumamoto 860-8556, Japan. Email: hdobaba@kumamoto-u.ac.jp.
}

Submitted Dec 02, 2020. Accepted for publication Jan 12, 2021.

doi: $10.21037 / \mathrm{hbsn}-20-820$

View this article at: http://dx.doi.org/10.21037/hbsn-20-820

We congratulate Dr. Dreyer and colleagues for their recently published study entitled "Precision oncology in surgery: Patient selection for operable pancreatic cancer" in Annals of Surgery (1). In this unique study, the authors tried to define preoperative clinical and molecular characteristics in order to improve patient selection for operable pancreatic cancer. Pancreatic ductal adenocarcinoma (PDAC) remains the most lethal type of human cancer due to its high chemoresistance and invasiveness (2). In resected PDAC, the median overall survival has increased from 22.1 to 35 months during the past 10 years, largely owing to improvements in adjuvant therapies $(3,4)$. On the other hand, the high recurrence rate even in patients who underwent curative resection, and chemoresistance in the recurrent sites to current systemic chemotherapies (FOLFIRINOX and nab-paclitaxel plus gemcitabine), are major issues in the treatment for unselected PDAC patient populations. Surgical resection offers the only chance of cure, but surgery can be associated with significant morbidity and decreased activity of daily life (ADL), especially in the Whipple procedure (pancreaticoduodenectomy). Recently, the importance of preoperative chemotherapy even in operable PDAC has been increasingly recognized (5). Although molecular markers are being used more frequently to select patients for systemic targeted agents, only imaging modalities are used to stage patients and to assess their suitability for operative resection. Decisions on primary surgery or a neoadjuvant approach are made without evaluation of biological measures of tumor aggressiveness, or of the risk of occult metastatic disease.

Dr. Dreyer and colleagues investigated the clinical impacts of aberrant expression of the calcium-binding proteins S100A2 and S100A4, using 3 independent PDAC cohorts (total participants $=1,184$ ), and they then developed a preoperative nomogram that incorporated S100A2 and S100A4 expression to predict postoperative survival. Among patients who were positive for both biomarkers, approximately $50 \%$ died within a year of resection. Indeed, these patients displayed the worst survival rates of only $54 \%$, $26 \%$, and $6 \%$ at 1,2 , and 5 years, respectively, compared to those who were negative for both biomarkers $(79 \%, 54 \%$, and $18 \%)$ or positive for one $(66 \%, 38 \%$, and $14 \%)$. Such nomograms, in combination with advances of precision medicine in surgery according to genetics and molecular biology using blood samples and preoperative tissue samples obtained by EUS-FNA (endoscopic ultrasound-fine needle aspiration), have the potential to improve selection for upfront surgery and neoadjuvant therapy, avoiding surgery in aggressive disease with occult micrometastases, and justifying more extensive resections in biologically favorable disease beyond the radiological assessment alone (Figure 1).

Recent gene expression studies in PDACs have identified subtypes with biological and prognostic relevance. By RNA sequencing and methylation arrays, hypomethylation of S100A2 was associated with the prognostic "squamous" subtype of PDAC (6). Likewise, in Dreyer et al.'s study, high S100A2 and positive S100A4 expression associated strongly with the squamous subtype in Bailey's classifications (1). Bailey et al. (6) categorized PDACs into four molecular subtypes based on the genetic mutations observed: squamous, pancreatic progenitor, immunogenic; and aberrantly differentiated endocrine exocrine (ADEX). The ranked prognostic relevance of each subtype for median 


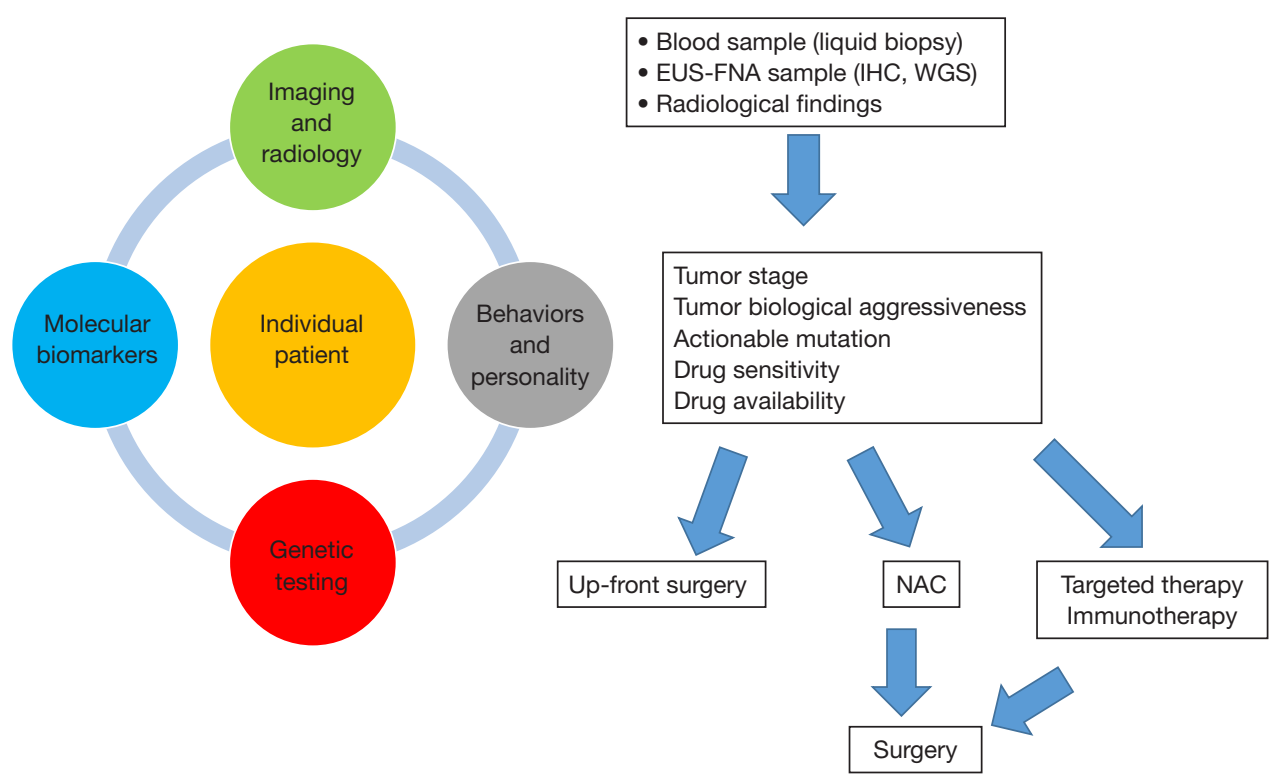

Figure 1 Precision medicine in surgery. IHC, immunohistochemistry; WGS, whole-genome sequencing; NAC, neoadjuvant chemotherapy.

survival time was immunogenic (30 months) $>$ pancreatic progenitor (25.6 months) > ADEX (23.7 months) > squamous (13.3 months). Collisson et al. (7) proposed classification of PDACs into three subtypes of classical, quasi-mesenchymal (QM), and exocrine-like. The survival of patients with the classical subtype following surgical resection and standard medical treatment was significantly better than that observed in patients with the QM subtype (ranked order, classical $>$ exocrine-like $>$ QM). Furthermore, they proposed a differential response to gemcitabine and erlotinib in the classical and QM subtypes in PDAC cell lines. Thus, this innovative system of reclassifying tumors and nomograms with subtype-specific molecular markers such as S100A2 and S100A4 could guide novel treatment decisions, because patients with a biologically high risk of early recurrence are unlikely to benefit from pancreatectomy and are better treated with neoadjuvant therapy for occult micrometastases. Other molecular markers associated with squamous type can be incorporated into the nomogram. We greatly appreciate Dreyer and colleagues' alternative approach toward precision surgery in pancreatic cancer.

Nevertheless, one major issue needs to be considered. The tumor marker CA19-9, which is the most widely used indicator of biological activity in PDAC, is not included in their nomogram because preoperative CA199 measurements were not available for a significant proportion of their cohort. Increased serum levels of CA199 have been shown to be associated with early recurrence and worse prognostic outcomes. Incorporating CA19-9 may thus improve the performance of a preoperative nomogram. In a recent study, a composite of 25 -gene signature from next-generation sequencing (NGS) in PDAC patients has been shown to identify patients with short- and long-term survival benefits after surgical resection of the primary tumor (8). NGS in exceptional responders may identify a subset of patients who would benefit from specific treatments rather than a surgical approach. There is an ongoing study (the COMPASS study) using real-time whole-genome sequencing and RNA sequencing to improve molecular and genetic characterization of PDAC and thus to help treatment selection for PDACs (9). Indeed, several molecular profiling studies have demonstrated that up to $30 \%$ of PDACs harbor actionable molecular alterations in DNA damage repair pathways (a determinant of sensitivity to platinum chemotherapy and PARP inhibitor) $(9,10)$.

A new era of precision medicine or surgery in pancreatic cancer is beginning. Nomogram and subtype classifications in PDACs need to be prospectively evaluated and to be used in the design of scientifically rational treatment strategies. Dreyer et al.'s study provides valuable information regarding not only preoperative nomograms with prognostic relevance but also precision surgery for PDAC.

\section{Acknowledgments}

Funding: None. 


\section{Footnote}

Provenance and Peer Review: This article was commissioned by the editorial office of Hepatobiliary Surgery and Nutrition. The article did not undergo external peer review.

Conflicts of Interest: Both authors have completed the ICMJE uniform disclosure form (available at https://hbsn. amegroups.com/article/view/10.21037/hbsn-20-820/coif). The authors have no conflicts of interest to declare.

Ethical Statement: The authors are accountable for all aspects of the work in ensuring that questions related to the accuracy or integrity of any part of the work are appropriately investigated and resolved.

Open Access Statement: This is an Open Access article distributed in accordance with the Creative Commons Attribution-NonCommercial-NoDerivs 4.0 International License (CC BY-NC-ND 4.0), which permits the noncommercial replication and distribution of the article with the strict proviso that no changes or edits are made and the original work is properly cited (including links to both the formal publication through the relevant DOI and the license). See: https://creativecommons.org/licenses/by-nc-nd/4.0/.

\section{References}

1. Dreyer SB, Pinese M, Jamieson NB, et al. Precision Oncology in Surgery: Patient Selection for Operable

Cite this article as: Hayashi $\mathrm{H}$, Baba $\mathrm{H}$. Precision surgery with the genetic assessment for operable pancreatic cancer beyond the radiological assessment alone. HepatoBiliary Surg Nutr 2021;10(2):261-263. doi: 10.21037/hbsn-20-820
Pancreatic Cancer. Ann Surg 2020;272:366-76.

2. Siegel RL, Miller KD, Jemal A. Cancer statistics, 2019. CA Cancer J Clin 2019;69:7-34.

3. Conroy T, Hammel P, Hebbar M, et al. FOLFIRINOX or Gemcitabine as Adjuvant Therapy for Pancreatic Cancer. N Engl J Med 2018;379:2395-406.

4. Uesaka K, Boku N, Fukutomi A, et al. Adjuvant chemotherapy of S-1 versus gemcitabine for resected pancreatic cancer: a phase 3, open-label, randomised, noninferiority trial (JASPAC 01). Lancet 2016;388:248-57.

5. Ye M, Zhang Q, Chen Y, et al. Neoadjuvant chemotherapy for primary resectable pancreatic cancer: a systematic review and meta-analysis. HPB (Oxford) 2020;22:821-32.

6. Bailey P, Chang DK, Nones K, et al. Genomic analyses identify molecular subtypes of pancreatic cancer. Nature 2016;531:47-52.

7. Collisson EA, Sadanandam A, Olson P, et al. Subtypes of pancreatic ductal adenocarcinoma and their differing responses to therapy. Nat Med 2011;17:500-3.

8. Birnbaum DJ, Finetti P, Lopresti A, et al. A 25-gene classifier predicts overall survival in resectable pancreatic cancer. BMC Med 2017;15:170.

9. Aung KL, Fischer SE, Denroche RE, et al. GenomicsDriven Precision Medicine for Advanced Pancreatic Cancer: Early Results from the COMPASS Trial. Clin Cancer Res 2018;24:1344-54.

10. Chantrill LA, Nagrial AM, Watson C, et al. Precision Medicine for Advanced Pancreas Cancer: The Individualized Molecular Pancreatic Cancer Therapy (IMPaCT) Trial. Clin Cancer Res 2015;21:2029-37. 